\title{
NORMAL STRUCTURE IN DUAL BANACH SPACES ASSOCIATED WITH A LOCALLY COMPACT GROUP
}

\author{
ANTHONY TO-MING LAU AND PETER F. MAH
}

\begin{abstract}
In this paper we investigated when the dual of a certain function space defined on a locally compact group has certain geometric properties. More particularly, we asked when weak* compact convex subsets in these spaces have normal structure, and when the norm of these spaces satisfies one of several types of Kadec-Klee property. As samples of the results we have obtained, we have proved, among other things, the following two results: (1) The measure algebra of a locally compact group has weak*-normal structure iff it has property SUKK* iff it has property SKK* iff the group is discrete; (2) Among amenable locally compact groups, the Fourier-Stieltjes algebra has property SUKK* iff it has property SKK* iff the group is compact. Consequently the Fourier-Stieltjes algebra has weak*-normal structure when the group is compact.
\end{abstract}

1. Introduction. In this paper we shall investigate when the dual of a certain function space defined on a locally compact group has certain geometric properties that proved to be useful in several areas of mathematics. To be more specific, we shall consider when weak* compact convex subsets in these spaces have normal structure, and when these spaces have the various types of Kadec-Klee property. It turns out that these properties are intimately related; and though quite distinct in general, they coincide in some of these spaces. We refer the reader to $[\mathbf{1 2}, \mathbf{2 1}]$ where similar problems were considered in Orlicz sequence spaces.

The concept of normal structure proved to be useful in the fixed point theory of nonexpansive maps. A central problem in the fixed point theory of nonexpansive maps is to determine those subsets of a Banach space which have the fixed point property for nonexpansive self-maps. A long standing problem was whether a weakly compact convex subset of a Banach space has the fixed point property for nonexpansive self-maps. With the appearance of Alspach's example [2] we now know there is a weakly compact convex set in $L_{1}[0,1]$ which need not have the fixed point property for nonexpansive self-maps. On the other hand, we know a weakly compact (weak* compact) convex subset in a (dual) Banach space does have the fixed point property for nonexpansive self-maps if the set has normal structure $[18,26]$.

Received by the editors September 1, 1987.

1980 Mathematics Subject Classification (1985 Revision). Primary 43A10, 43A15, 43A60, 43A77, 22D15, 46B05, 46B20; Secondary 47H10, 47D35.

Key words and phrases. Locally compact group, amenable group, measure algebra, left uniformly continuous functions, (weakly) almost periodic functions, Fourier-Stieltjes algebra, weak*normal structure, Kadec-Klee property, uniformly Kadec-Klee property.

Both authors' research were supported by NSERC of Canada. 
Norms that satisfy a type of Kadec-Klee property (the precise definition of the various types is given in $\S 2$ ) appear in diverse areas of mathematics. For example, it appears in some proofs of theorems on renorming, as in the Kadec-Klee-Asplund Renorming Theorem [9], [8, p. 146]. The terminologies in the literature are not yet standardized. For example, in [3] the Kadec-Klee property is known as the Radon-Riesz property, presumably in honor of the Radon-Riesz Theorem, which states that the spaces $L_{p}, 1<p<\infty$, have this property. In abstract harmonic analysis, a property similar to the Kadec-Klee property, but for nets, defines the $w \sim$-topology in $[\mathbf{1}, \mathbf{2 2}]$, whereas in $[\mathbf{1 5}]$, it is called the $\tau_{n w^{*}}$ topology. This property is also known as property $(* *)$ in [29]. A stronger uniform version of the KadecKlee property was introduced and studied by Huff [17], while van Dulst and Sims considered the corresponding notion for dual spaces. Van Dulst and Sims showed that both of these properties are strong enough to yield normal structure for either weakly or weak* compact convex subsets.

The organization of this paper is as follows. $\S 2$ is devoted to definitions and notations. The definitions of weak-, weak*-normal structure, and the various types of Kadec-Klee property alluded to earlier are given, and the relationships among them are discussed. In $\S 3$, we determine when the following dual spaces associated with a locally compact group have weak*-normal structure, or one of the types of Kadec-Klee property: the measure algebra on the group, the duals of any $C^{*}$ subalgebra of weakly almost periodic functions that contains the constants and the functions that vanish at infinity, the left uniformly continuous functions, and the almost periodic functions. In $\S 4$, our main result is Theorem 5 , which states that among amenable locally compact groups, the Fourier-Stieltjes algebra $B(G)$ has property $\mathrm{SUKK}^{*}$ iff it has property $\mathrm{SKK}^{*}$ iff $G$ is compact. Consequently, when $G$ is compact, $B(G)$ has weak*-normal structure. Our technique of proof for some part of this theorem was inspired by a recent result of Chris Lennard [25]. We have used some of his ideas in concocting a proof that works for nets as well as for a variety of spaces, namely the dual of any $C^{*}$-algebra with a bounded approximate identity consisting of projections of finite rank. This is embodied in our Lemma 4.

Finally, it is a pleasure for us to thank Chris Lennard for sending us a preprint of his work.

2. Definitions and notations. Let $K$ be a bounded closed convex subset of a Banach space $E$. A point $x$ in $K$ is called a diametral point of $K$ if

$$
\sup \{\|x-y\|: y \in K\}=\operatorname{diam}(K)
$$

where $\operatorname{diam}(K)$ denotes the diameter of $K$. The set $K$ is said to have normal structure if every nontrivial convex subset (i.e., contains at least two points) $H$ of $K$ contains a nondiametral point of $H$. A Banach space has weak-normal structure if every nontrivial weakly compact convex subset has normal structure. If the Banach space is also a dual space then it has weak*-normal structure if every nontrivial weak* compact convex subset has normal structure. It is clear that a dual Banach space has weak-normal structure whenever it has weak*-normal structure.

A [dual] Banach space $E$ is said to have the fixed point property (FPP) [(FPP*)] if for every weakly [weak ${ }^{*}$ ] compact convex subset $K$ of $E$ and for every nonexpansive $T: K \rightarrow K, T$ has a fixed point in $K$. Kirk proved that if $E$ has weak-normal 
structure then $E$ has property FPP [18]. Subsequently, Lim proved that a dual Banach space has property FPP* whenever it has weak*-normal structure [26].

The following types of Kadec-Klee properties satisfied by a norm will be considered.

(a) A Banach space $E$ is said to have the Kadec-Klee property (KK) if whenever $\left(x_{n}\right)$ is a sequence in the unit ball of $E$ that converges weakly to $x$, and $\operatorname{sep}\left(\left(x_{n}\right)\right)>$ 0 , where

$$
\operatorname{sep}\left(\left(x_{n}\right)\right) \equiv \inf \left\{\left\|x_{n}-x_{m}\right\|: n \neq m\right\},
$$

then $\|x\|<1$. This property, given in different form, is known as property $(\mathrm{H})$ in [8], property (A) in [7], or the Radon-Riesz property in [2]. The definition as given above is due to Huff [17].

(b) A Banach space $E$ is said to have the uniformly Kadec-Klee property (UKK) if for every $\varepsilon>0$ there is a $0<\delta<1$ such that whenever $\left(x_{n}\right)$ is a sequence in the unit ball of $E$ converging weakly to $x$ and $\operatorname{sep}\left(\left(x_{n}\right)\right)>\varepsilon$ then $\|x\| \leq \delta$. This property was introduced by Huff [17], who showed that property UKK is strictly stronger than property KK. Van Dulst and Sims showed that a Banach space with property UKK has property FPP [11].

Corresponding to each of the above properties there is a stronger property defined by replacing the sequence in the above definitions by a net. We call these properties by the strong Kadec-Klee property (SKK) and the strong uniformly Kadec-Klee property (SUKK). For dual Banach spaces we have the corresponding properties involving the weak* topology, which we denote by $\mathrm{KK}^{*}, \mathrm{SKK}^{*}, \mathrm{UKK}^{*}$ and SUKK* First, properties $\mathrm{KK}^{*}$ and $\mathrm{SKK}^{*}$ are defined by replacing weak convergence by weak* convergence in the definition of properties KK and SKK, respectively. Property $\left(\mathrm{SKK}^{*}\right)$ is just property $(* *)$ in [29]. It is known that a dual space which is locally uniformly convex has property $\mathrm{SKK}^{*}$ and that a space with property SKK* has the Radon-Nikodym property [29].

It is natural to define a property similar to UKK by replacing the weak convergence by weak* convergence in UKK and calling it UKK*. However, van Dulst and Sims found the following definition is more useful.

(c) A dual Banach space $E$ has property $\mathrm{UKK}^{*}$ if for every $\varepsilon>0$ there is a $0<\delta<1$ such that whenever $A$ is a subset of the closed unit ball of $E$ containing a sequence $\left(x_{n}\right)$ with $\operatorname{sep}\left(\left(x_{n}\right)\right)>\varepsilon$ then there is an $x$ in weak ${ }^{*}$-closure $(A)$ such that $\|x\| \leq \delta$.

They proved that a dual Banach space with property UKK* has property FPP* [11]. Moreover, they observed that if the dual unit ball is weak* sequentially compact then property $\mathrm{UKK}^{*}$, as defined above, is equivalent to the condition obtained from UKK by replacing weak convergence by weak* convergence. Finally, we define property SUKK* as follows:

(d) A dual Banach space $E$ has property SUKK* if for every $\varepsilon>0$ there is a $0<\delta<1$ such that whenever $\left(x_{\alpha}\right)$ is a net is the unit ball of $E$ that converges to $x$ in the weak ${ }^{*}$ topology and $\operatorname{sep}\left(\left(x_{\alpha}\right)\right)>\varepsilon$ then $\|x\| \leq \delta$. It is easy to see that property SUKK* implies property UKK*.

We summarize the relationships among the various concepts in the following diagram. We shall denote weak- (weak*-) normal structure by w.n.s. (w*.n.s.) and quasi-weak- (weak*-) normal structure by q.w.n.s. (q.w*.n.s.). (See $[24]$ for the definitions.) It is understood that for some comparison to make sense we assume 
that we are in a dual space.

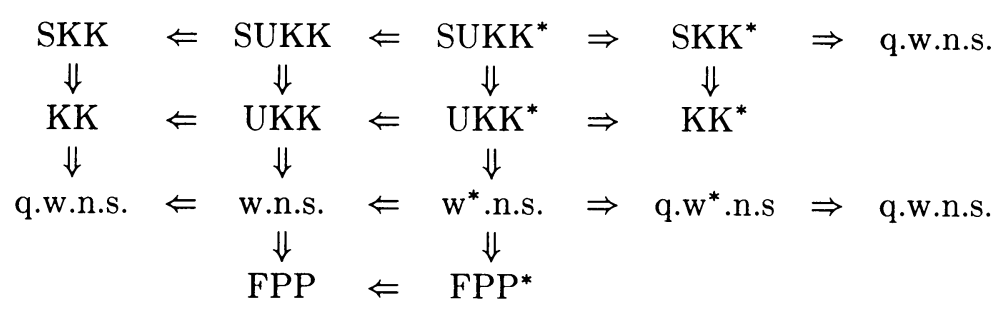

We now mention some examples which show that, generally, some of the above implications cannot be reversed.

(1) Let $X=\left(l_{2} \oplus l_{3} \oplus \cdots \oplus l_{n} \oplus \cdots\right)_{2}$. Then, as noted by Huff [17], $X$ is reflexive, has property KK but not UKK. Moreover, we observe that $X$ is separable, and being reflexive, its unit ball is weakly (or weak*) metrizable. Thus in $X$ we have $\mathrm{SKK}^{*} \Leftrightarrow \mathrm{KK}^{*} \Leftrightarrow \mathrm{SKK} \Leftrightarrow \mathrm{KK}$, and $\mathrm{SUKK}^{*} \Leftrightarrow \mathrm{UKK}^{*} \Leftrightarrow \mathrm{SUKK} \Leftrightarrow \mathrm{UKK}$. So this same example shows that SKK* or SKK does not imply SUKK or SUKK*.

(2) Let $l_{2}$ be renormed according to [32]. Then, as proved by Smith and Turett, $l_{2}$ with this new norm is reflexive and locally uniformly rotund but does not have normal structure. Since the space is reflexive, normal structure is equivalent to weak-normal structure as well as weak*-normal structure. On the other hand, since the space is locally uniformly rotund, it has property $\mathrm{SKK}^{*}$. Consequently we have a space that has property $\mathrm{SKK}^{*}$ (or SKK) but not weak*- (or weak-) normal structure. Note also we have an example of a space with quasi-weak*normal structure but not weak*-normal structure.

(3) We will give an example of a space with property SUKK but not KK* after we prove Theorem 2 .

3. Normal structure in $M(G)$ and other dual spaces. Let $X$ be a locally compact Hausdorff space, and $C(X)$ be the space of bounded continuous complexvalued functions defined on $X$ with the supremum norm. Let $C_{0}(X)$ be the subspace of $C(X)$ consisting of functions "vanishing at infinity," and $M(X)$ be the space of bounded regular Borel measure on $X$, with the variation norm. Let $M_{d}(X)$ be the subspace of $M(X)$ consisting of the discrete measures on $X$. It is well known that the dual of $C_{0}(X)$ can be identified with $M(X)$, and that $M_{d}(X)$ is isometrically isomorphic to $l_{1}(X)$. See $[\mathbf{1 6}]$.

Recall that a topological space $X$ is scattered (or dispersed) if $X$ does not contain any perfect subset. For more information on scattered spaces we refer the reader to $[31]$.

LEMMA 1. Let $X$ be a locally compact Hausdorff space. Then the following statements are equivalent:

(1) $X$ is scattered.

(2) $M(X)=M_{d}(X)$.

(3) $M(X)$ is isometrically isomorphic to $l_{1}(X)$.

(4) $M(X)$ has property SUKK.

(5) $M(X)$ has property UKK.

(6) $M(X)$ has weak-normal structure.

(7) $M(X)$ has property FPP. 
Proof. (1) $\Rightarrow(2)$. The proof is similar to the case when $X$ is compact, and so we omit the details. See $[\mathbf{2 0}$, p. 52$]$.

$(2) \Rightarrow(3)$ follows from [16, p. 270].

(3) $\Rightarrow(4)$. Let $0<\varepsilon<2$. Let $\left(\varphi_{\alpha}\right)$ be a net in the unit ball of $M(X)$ that converges to $\varphi$ weakly, and $\operatorname{sep}\left(\left(\varphi_{\alpha}\right)\right)>\varepsilon$. We will prove that $\|\varphi\|<1-\varepsilon / 2+\eta$ for all $\eta>0$. So let $\eta$ be arbitrary. Let $\psi_{\alpha}$ and $\psi$ be the images of $\varphi_{\alpha}$ and $\varphi$, respectively, under the isometric isomorphism $\Phi$ from $M(X)$ onto $l_{1}(X)$. Then $\psi_{\alpha}$ converges to $\psi$ weakly in $l_{1}(X)$. Now the $l_{1}$ norm satisfies

$$
\left\|\psi_{\alpha}\right\| \geq\left\|\psi_{\alpha}-\psi\right\|-\|\psi\|+2 \sum_{s \in \sigma}\left(|\psi(s)|-\left|\left(\psi_{\alpha}-\psi\right)(s)\right|\right)
$$

for any subset $\sigma$ of $X$. Since $\operatorname{sep}\left(\left(\psi_{\alpha}\right)>\varepsilon\right.$ we may assume that $\left\|\psi_{\alpha}-\psi\right\|>\varepsilon / 2$ for all $\alpha$. Now let $\sigma$ be a finite subset of $X$ such that

$$
\sum_{s \in \sigma}|\psi(s)|>\|\psi\|-\eta / 4
$$

For this $\sigma$ we can use the weak convergence of $\psi_{\alpha}-\psi$ to 0 to choose an $\alpha_{0}$ so that for all $\alpha \geq \alpha_{0}$ we have

$$
\sum_{s \in \sigma}\left|\psi_{\alpha}(s)-\psi(s)\right|<\eta / 4
$$

Then for all $\alpha \geq \alpha_{0}$ we have from $(*)$,

$$
1 \geq\left\|\psi_{\alpha}-\psi\right\|-\|\psi\|+2\|\psi\|-\eta / 2-\eta / 2 \geq \varepsilon / 2+\|\psi\|-\eta .
$$

Thus we have $\|\varphi\|=\|\psi\|<1-\varepsilon / 2+\eta$. This proves that $M(X)$ has property SUKK.

(4) $\Rightarrow(5)$. Statement (4) is formally stronger than (5).

$(5) \Rightarrow(6)$. This is proved by van Dulst and Sims [11].

(6) $\Rightarrow(7)$. This is well known.

(7) $\Rightarrow(1)$. If $X$ is not scattered then $X$ contains a perfect set $Y$. Since $Y$ is closed, it is locally compact and so it is a $G_{\delta}$-subset of its Stone-Čech compactification $\beta Y$ [13, p. 251]. Since $Y$ does not contain any isolated points, it follows from Theorem 3(i) in [19] that there is a nonzero nonatomic regular Borel measure $\mu^{*}$ on $Y$. This $\mu^{*}$ can be used to generate a nonatomic regular Borel measure $\mu$ on $X$ by the process described in [16, (11.45), p. 133]. By [16, Theorem 14.17, p. 176] there is an isometric isomorphic copy of $L_{1}(X, \mu)$ in $M(X)$. According to [20, Corollary, p. 136], $L_{1}(X, \mu)$ contains an isometric isomorphic copy of $L_{1}[0,1]$. Now let $K$ be the weakly compact convex subset of $L_{1}[0,1]$ and $T$ be the nonexpansive map on $K$ as constructed by Alspach [2]. Clearly $K$ (its image in $M(X)$ ) is also weakly compact in $M(X)$. Thus $M(X)$ does not have property FPP, contradicting (7). So (1) follows.

COROLlary 1. Let $X$ be a compact Hausdorff space. Then $M(X)$ has weak*normal structure if and only if $X$ is finite.

Proof. $M(X)$ has weak*-normal structure, then $M(X)$ must have weak-normal structure. So by Lemma $1, M(X)=M_{d}(X)$. Let $K=\{\mu \in M(X): \mu \geq 0$ and $\|\mu\|=1\}$. Then $K$ is a weak*-compact convex subset of $M(X)$, and $\operatorname{diam}(K)=2$. By assumption, there exists $\mu_{0} \in K$ such that $\sup \left\{\left\|\mu-\mu_{0}\right\|: \mu \in K\right\}=\lambda<2$. 
Write $\mu=\sum_{i=1}^{\infty} \lambda_{i} \delta_{x_{i}}$, where $\delta_{x_{i}}$ is the Dirac measure at $x_{i} \in X, \lambda_{i}>0$, and $\sum_{i=1}^{\infty} \lambda_{i}<\infty$. Let $0<\varepsilon<2-\lambda$. Choose $N$ so large such that $\sum_{i=N+1}^{\infty} \lambda_{i}<\varepsilon / 2$. Then

$$
\begin{aligned}
\left\|\mu-\delta_{x_{N+1}}\right\| & =\left\|\sum_{i=1}^{N} \lambda_{i} \delta_{x_{i}}-\delta_{x_{N+1}}+\sum_{i=N+1}^{\infty} \lambda_{i} \delta_{x_{i}}\right\| \\
& \geq\left\|\sum_{i=1}^{N} \lambda_{i} \delta_{x_{i}}-\delta_{x_{N+1}}\right\|-\sum_{i=N+1}^{\infty} \lambda_{i} \geq 2-\varepsilon>\lambda,
\end{aligned}
$$

which is impossible.

REMARK. Let $X$ denote the one point compactification of the positive integers $N$. Then $X$ is scattered and hence $C(X)^{*}$ is isometrically isomorphic to $l_{1}(X)$. But $l_{1}(X)$ does not have weak*-normal structure when considered as the dual space of $C(X)$ even though $l_{1}(X)$ has weak*-normal structure when regarded as the dual space of $c_{0}(X)[26]$. This example shows that one direction of our Theorem 4 in [24] (i.e., if $C(X)^{*}$ is isometrically isomorphic to $l_{1}(\Gamma)$ for some nonempty set $\Gamma$, then $C_{0}(X)^{*}$ satisfies Lim's condition) is incorrect. However, the proof for this direction is correct when the isomorphism is weak*-weak* continuous.

In what follows let $G$ be a locally compact group. For each $x \in G$, define the left translation operator $l_{x}$ on $C(G)$ by $l_{x} f(y)=f(x y)$. Similarly a right translation operator $r_{x}$ can be defined. Sometimes it is convenient for us to denote $l_{x} f\left(r_{x} f\right)$ by ${ }_{x} f\left(f_{x}\right)$. For $f \in C(G)$, the left orbit of $f$ is defined by $L O(f)=\left\{{ }_{x} f: x \in G\right\}$. Similarly we can define the right orbit $R O(f)$ of $f$. We recall the definitions of the following function spaces defined on $G$ :

(a) $L U C(G)=\left\{f \in C(G)\right.$ : the map $x \rightarrow{ }_{x} f, x \in G$, is continuous when $C(G)$ has the norm topology $\}$. This is the space of left uniformly continuous functions (or the right uniformly continuous functions in the language of [16]) on $G$.

(b) $W A P(G)=\{f \in C(G): L O(f)$ is relatively weakly compact in $C(G)\}$. As is well known $f \in W A P(G)$ iff $R O(f)$ is relatively weakly compact.

(c) $A P(G)=\{f \in C(G): L O(f)$ is relatively norm compact $\}$. It is known that $f \in A P(G)$ iff $R O(f)$ is relatively norm compact. Functions in $W A P(G)(A P(G))$ are called weakly almost periodic (almost periodic). For further information we refer the reader to [4].

LEMMA 2. Let $G$ be a locally compact group and $X$ be a closed subspace of $C(G)$ containing $C_{0}(G)$. If $X^{*}$ has the property that weak* convergence and weak convergence for sequences agree on the unit sphere then $G$ is discrete.

ProOF. Let $\lambda$ denote the (left) Haar measure on $G$, and let $e$ be the identity of $G$. If $G$ is not discrete then $\lambda(e)=0$. Let $U_{n}$ be a sequence of open relatively compact sets containing $e$ such that $\lambda\left(U_{n}\right) \rightarrow 0$.

Define a sequence $\left(h_{n}\right)$ of functions in $C_{0}(G)$ as follows. Let $h_{1}$ be defined by

$$
h_{1}(x)= \begin{cases}1 & \text { if } x=e, \\ 0 & \text { if } x \in G \sim U_{1},\end{cases}
$$

and $0 \leq h_{1}(x) \leq 1$ for all $x$ in $G$. Let $V_{1}=\left\{y: h_{1}(y) \neq 0\right\}$. Then clearly $V_{1} \subset U_{1}$. Define $h_{2}$ by

$$
h_{2}(x)= \begin{cases}1 & \text { if } x=e \\ 0 & \text { if } x \in G \sim\left(U_{2} \cap V_{1}\right)\end{cases}
$$


and $0 \leq h_{2}(x) \leq 1$ for all $x$ in $G$. Let $V_{2}=\left\{y: h_{2}(y) \neq 0\right\}$. Then $V_{2} \subset U_{1} \cap V_{1}$. In general, if $h_{n}$ has been defined, we let $V_{n}=\left\{y: h_{n}(y) \neq 0\right\}$. Then we define $h_{n+1}$ by

$$
h_{n+1}(x)= \begin{cases}1 & \text { if } x=e, \\ 0 & \text { if } x \in G \sim\left(U_{n+1} \cap V_{n}\right),\end{cases}
$$

and $0 \leq h_{n}(x) \leq 1$ for all $x$ in $G$.

Now for each $n$ define a function $d_{n}$ on $G \times G$ by $d_{n}(x, y)=\left\|l_{x} h_{n}-l_{y} h_{n}\right\|$. Then it can be verified that each $d_{n}$ is a continuous pseudo-metric on $G$ and that

$$
K_{n}=\left\{x \in G: d_{n}(x, e)=0\right\}
$$

is a compact subgroup of $G$. Furthermore, if $K=\bigcap_{n=1}^{\infty} K_{n}$, then $G / K$ is metrizable. Notice that we have

(i) $K \subset V_{n}$ for each $n$.

(ii) $V_{n}$ is decreasing, $e \in V_{n}$, and $V_{n} \subset U_{n}$ for each $n$.

(iii) $\lambda\left(V_{n}\right) \rightarrow 0$.

Let $W_{n}$ be a decreasing sequence of relatively compact neighbourhoods of $K$ such that $K=\bigcap_{n=1}^{\infty} W_{n}$. Such $W_{n}$ exist because $G / K$ is metrizable. Let $\varphi_{n}=$ $\left(1 / \lambda\left(W_{n}\right)\right) 1_{W_{n}}$. Then $\varphi_{n} \in L_{1}(G)$, and for every $f \in C(G)$, we have $\varphi_{n}(f)=$ $\int f \varphi_{n} d \lambda \rightarrow \int f d \lambda_{K}$, where $\lambda_{K}$ is the normalized Haar measure on $K$. It follows then $\varphi_{n}$ converges to $\lambda_{K}$ in the weak* topology of $X^{*}$, and by assumption, it must also converge in the weak topology. Since the weak topology in $X^{*}$ is the relative weak topology for a subset of $M(G)$ it follows that the sequence converges also in the weak topology in $M(G)$. Since $L_{1}(G)$ is weakly sequential complete, we have $\lambda_{K} \in L_{1}(G)$. This is a contradiction since $\lambda_{K}(K)=1$ while $\lambda(K)=0$.

THEOREM 1. Let $G$ be a locally compact group. Then the following statements are equivalent.

(1) $G$ is discrete.

(2) $M(G)$ is isometrically isomorphic to $l_{1}(G)$.

(3) $M(G)$ has property SUKK*.

(4) $M(G)$ has property UKK*.

(5) $M(G)$ has property SKK*.

(6) $M(G)$ has property $\mathrm{KK}^{*}$.

(7) Weak* convergence and weak convergence of sequences agree on the unit sphere of $M(G)$.

(8) $M(G)$ has weak* normal structure.

(9) $M(G)$ has property FPP*.

PROOF. The equivalence of (1) and (2) follows from [16, Theorem 19.15, p. 270] and Lemma 1.

$(2) \Rightarrow(3)$. The proof is similar to the proof of $(3) \Rightarrow(4)$ in Lemma 1 , and so we omit the details. The difference in the proofs is that now we can use the fact that $G$ is discrete to prove $\psi_{\alpha}-\psi$ converges to 0 in the weak* topology and so inequality $(* *)$ in the proof of Lemma 1 follows.

The implications (3) $\Rightarrow(5) \Rightarrow(6)$ and $(3) \Rightarrow(4) \Rightarrow(6)$ are obvious.

$(6) \Rightarrow(7)$. This is obvious since property $\mathrm{KK}^{*}$ is equivalent to the statement that norm convergence and weak* convergence of sequences agree on the unit sphere.

(7) $\Rightarrow(1)$. This follows from Lemma 2 . 
$(4) \Rightarrow(8) \Rightarrow(9)$ is proved by van Dulst and Sims [11].

$(9) \Rightarrow(2)$. If $M(G)$ has property FPP* then it has property FPP and so by Lemma $1,(2)$ follows.

LEMMA 3. Let $G$ be a locally compact group and let $N$ be a $C^{*}$-subalgebra of $C(G)$ containing $C_{0}(G)$.

(i) If $N^{*}$ has weak-normal structure then $G$ is discrete.

(ii) If $N$ contains the constants and $N^{*}$ has weak*-normal structure then $G$ is finite.

PROOF. (i) If $G$ is not discrete, let $\mu \in L_{1}(G)$, and let $\mu^{\sim}(f)=\int f d \mu$. Since $N$ is isometrically isomorphic to $C_{0}(\Delta)$, where $\Delta$ is the spectrum of $N$, and since $N$ has weak-normal structure, it follows from Lemma 1 that $\mu^{\sim}=\sum_{i=1}^{\infty} \lambda_{i} \varphi_{i}^{\sim}$, where $\varphi_{i}^{\sim} \in \Delta, \lambda_{i} \in \mathbf{C}, \sum_{i=1}^{\infty}\left|\lambda_{i}\right|<\infty$. Thus $\mu=\sum_{i=1}^{\infty} \lambda_{i} \varphi_{i}$, where $\varphi_{i}$ is the restriction of $\varphi_{i}^{\sim}$ to $C_{0}(G)$. But for each $i \varphi_{i}=\delta_{g_{i}}, g_{i} \in G$ and so $\mu=\sum_{i=1}^{\infty} \lambda_{i} \delta_{g_{i}} \in l_{1}(G)$, which is impossible.

(ii) If $N^{*}$ has weak*-normal structure and $N$ contains the constants then $N$ is isometrically isomorphic to $C(\Delta), \Delta$ the spectrum of $N$. By Corollary $1, \Delta$ must be finite. Since $N$ contains $C_{0}(G)$, the natural embedding $x \rightarrow \delta_{x}$ of $G$ into $\Delta$ is 1-1. So $G$ must be finite.

THEOREM 2. Let $G$ be a locally compact group. Let $N$ be a $C^{*}$-subalgebra of $W A P(G)$ containing $C_{0}(G)$ and the constants. Then the following statements are equivalent:

(1) $G$ is finite.

(2) $N^{*}$ has property SUKK*.

(3) $N^{*}$ has property SKK*.

(4) $N^{*}$ has property $\mathrm{UKK}^{*}$.

(5) $N^{*}$ has property $\mathrm{KK}^{*}$.

(6) Weak* convergence and weak convergence for sequences agree on the unit sphere of $N^{*}$.

(7) $N^{*}$ has weak ${ }^{*}$-normal structure.

ProOF. The implications $(1) \Rightarrow(2) \Rightarrow(3) \Rightarrow(5) \Rightarrow(6)$ and $(2) \Rightarrow(4)$ are clear. That $(4) \Rightarrow(7)$ is a result of van Dulst and Sims $[\mathbf{1 1}]$, while $(7) \Rightarrow(1)$ follows from Lemma 3 . It remains to prove $(6) \Rightarrow(1)$. Now by Lemma $2, G$ must be discrete. If $G$ is not finite, let $G_{0}$ be a countable subgroup of $G$ generated by countably many elements of $G$. Consider the restriction map $\rho: W A P(G) \rightarrow W A P\left(G_{0}\right)$ defined by $\rho f=\left.f\right|_{G_{0}}$. Then $\rho f \in W A P\left(G_{0}\right)$ [28, Theorem 1.8]. Furthermore, $\rho$ is onto since for each $g \in W A P\left(G_{0}\right)$, the function

$$
g^{\sim}(x)= \begin{cases}g(x) & \text { if } x \in G_{0} \\ 0 & \text { otherwise. }\end{cases}
$$

is in $W A P(G)[28$, Theorem 2]. We now use an idea by Lau and Losert [23]. By [27, Corollary p. 74] there exists a sequence $\varphi_{n}=\sum_{i=1}^{m} \lambda_{i}^{n} \delta_{a_{i}}, a_{i} \in G_{0}, \lambda_{i}^{n}>0$, $\sum_{i=1}^{m} \lambda_{i}^{n}=1$, such that $\varphi_{n}(f)$ converges to $m(f)$ for each $f$ in $W A P\left(G_{0}\right)$, where $m$ is the unique invariant mean on $W A P\left(G_{0}\right)$. Let $\varphi_{n}^{\prime}=\left.\rho^{*}\left(\varphi_{n}\right)\right|_{N}$ and $m^{\prime}=\left.\rho^{*}(m)\right|_{N}$. Then, as readily checked, $\varphi_{n}^{\prime}$ converges to $m^{\prime}$ in the weak* topology of $N^{*}$ and $\left\|\varphi_{n}^{\prime}\right\|=\left\|m^{\prime}\right\|=1$. So by assumption, $\varphi_{n}^{\prime}$ converges to $m^{\prime}$ in the weak topology 
of $N^{*}$. Now note that for each $a \in G_{0}, \rho^{*}\left(\delta_{a}\right)=\delta_{a}^{\prime}$ is the point evaluation at $a$ on $N$. So if $K$ is the convex hull of $\left\{\delta_{a}^{\prime}: a \in G_{0}\right\}$ then it follows that $m^{\prime}$ is in the weak-closure of $K$. By Mazur's theorem, there exists a sequence $\psi_{n}^{\prime}$ in $K$ such that $\left\|\psi_{n}^{\prime}-m^{\prime}\right\| \rightarrow 0$. Denote by \|\|$\quad\|\|_{1}$ and \|\|\|\|$_{0}$ the norms on $C_{0}(G)^{*}$ and $C_{0}\left(G_{0}\right)^{*}$, respectively. By regarding $\psi_{n}^{\prime}$ and $m^{\prime}$ as elements in $C_{0}(G)^{*}$, we have $\left\|\psi_{n}^{\prime}-m^{\prime}\right\|_{1} \rightarrow 0$ as well. Since $\rho$ maps $C_{0}(G)$ onto $C_{0}\left(G_{0}\right)$, we must also have $\left\|\mid \psi_{n}-m\right\|_{0} \rightarrow 0$, where $\psi_{n}$ denotes the corresponding convex combination of elements in $\left\{\delta_{a}: a \in G_{0}\right\}$. But $\left\|\left|\psi_{n}\right|\right\|_{0}=1$ and $\|m\|_{0}=0[\mathbf{4}$, p. 41], which is impossible. So $G$ must be finite.

REMARK. Let $G$ be an infinite discrete group, and let $N$ be the closed $C^{*}$ subalgebra generated by $C_{0}(G)$ and the constants. Then $N$ is isometrically isomorphic to $C(\Delta)$, where $\Delta$ is the one-point compactification of $G$. Since $\Delta$ is scattered, it follows from Lemma 1 that $N^{*}$ has property SUKK. However $N^{*}$ does not have property $\mathrm{KK}^{*}$ by Theorem 2 . Thus SUKK does not imply $\mathrm{KK}^{*}$.

THEOREM 3. Let $G$ be a locally compact group. Then

(1) Weak* convergence and weak convergence for sequences agree on the unit sphere of $L U C(G)^{*}$ iff $G$ is discrete.

(2) $L U C(G)^{*}$ has weak ${ }^{*}$-normal structure iff $G$ is finite.

Proof. (1) The necessity follows from Lemma 2. For the sufficiency, if $G$ is discrete then $\operatorname{LUC}(G)=l_{\infty}(G)$, and the result then follows from a result of Grothendieck [10, Theorem 15, p. 103].

For (2), one part is trivial while the other is a consequence of Lemma 3.

THEOREM 4. Let $G$ be a locally compact group. Then $A P(G)^{*}$ has weak*normal structure iff $A P(G)$ is finite dimensional.

Proof. Let $G^{a}$ denote the almost periodic compactification of $G$. As known $A P(G)$ is isomorphic to $C\left(G^{a}\right)$ and $G^{a}$ is a compact group. Apply now Lemma 3.

4. Normal structure in $B(G)$. Let $\mathscr{H}$ be a Hilbert space, and $\mathscr{T}(\mathscr{H})$ be the space of trace-class operators on $\mathscr{H}$. Recently Lennard [25] showed that $\mathscr{T}(\mathscr{H})$ has property UKK*. Consequently, $\mathscr{T}(\mathscr{H})$ has weak*-normal structure. This answers affirmatively a question raised by the authors in [24]. In this section we show that there is a class of $C^{*}$-algebra whose dual has property $\left(\mathrm{UKK}^{*}\right)$, from which we can recover Lennard's result. In fact, we can prove that this class of $C^{*}$-algebra has the stronger property SUKK* ${ }^{*}$. This generalization allows us to prove the main result in this section, namely the Fourier-Stieltjes algebra $B(G)$, when $G$ is a compact group, has property SUKK* and hence, the weak*-normal structure, when regarded as the dual of the group $C^{*}$-algebra $C^{*}(G)$. It also improves, at least formally, a result of Granirer and Leinert [15], who proved that $B(G)$ has property SKK* when $G$ is compact. Moreover, we shall prove that among amenable locally compact groups (see [30] for the definition of amenable locally compact group), $B(G)$ has SUKK* iff it has SKK* iff $G$ is compact.

Notice that when $G$ is an abelian locally compact group, then $B(G) \cong M(\hat{G})$ and $C^{*}(G) \cong C_{0}(\hat{G})$, where $\hat{G}$ is the dual group of $G$. It follows from Theorem 1 that $B(G)$ has the weak*-normal structure iff $\hat{G}$ is discrete, or equivalently, $G$ is compact. However, we do not know in the nonabelian case whether compact groups are the only ones for which $B(G)$ has the weak*-normal structure. 
LEMMA 4. Let $\mathscr{A}$ be a $C^{*}$-algebra with a bounded approximate identity $\left\{P_{\alpha}\right\}$ consisting of projections of finite rank. Then $\mathscr{A}^{*}$ has property SUKK*.

ProOF. Suppose $\mathscr{A}^{*}$ does not have property SUKK*. Then there exists an $\varepsilon_{0}>0$ such that for every $0<\delta<1$, there is a net $\left(\varphi_{\alpha}\right)$ and $\varphi$ in the closed unit ball with $\operatorname{sep}\left(\left(\varphi_{\alpha}\right)\right)>\varepsilon_{0}$, and converging to $\varphi$ in the weak* topology but $\|\varphi\|>\delta$. Now let $\varepsilon_{1}<\varepsilon_{0}, 0<\varepsilon_{1}<1$ and pick $0<\delta_{0}<1$ so that $\delta_{0}>\left(1-\varepsilon_{1}^{2} / 12\right)^{1 / 2}$. Pick the net $\left(\varphi_{\alpha}\right)$ and $\varphi$ with the properties indicated above corresponding to $\delta=\delta_{0}$. Let $X_{0} \in \mathscr{A},\left\|X_{0}\right\|=1$, be a weak* linear functional which separates $\varphi$ and the closed ball of radius $\delta_{0}$ centred at 0 . Denote the slice $\left\{\varphi \in \mathscr{A}^{*}:\|\varphi\| \leq 1, \operatorname{Re}\left\langle X_{0}, \varphi\right\rangle \geq \delta_{0}\right\}$ by $S\left[X_{0}, \delta_{0}\right]$. Since $\left(\varphi_{\alpha}\right)$ converges to $\varphi$ in the weak* topology the net $\left(\varphi_{\alpha}\right)$ is eventually in $S\left[X_{0}, \delta_{0}\right]$. Since this subnet also has separation exceeding $\varepsilon_{0}$ we may assume the original net is contained in $S\left[X_{0}, \delta_{0}\right]$.

Now for each $\psi \in S\left[X_{0}, \delta_{0}\right]$, define, for each $\tau$,

$$
\alpha_{\tau}(\psi)=\left\|P_{\tau} \psi Q_{\tau}\right\|+\left\|Q_{\tau} \psi P_{\tau}\right\|+\left\|Q_{\tau} \psi Q_{\tau}\right\|
$$

with $Q_{\tau}=I-P_{\tau}$, where $I$ is the identity in the enveloping von Neumann algebra $\mathscr{A}^{* *}$ of $\mathscr{A}$. We first show that

$$
\alpha_{\tau}(\psi) \leq 3^{1 / 2}\left(1-\left\|P_{\tau} \psi P_{\tau}\right\|^{2}\right)^{1 / 2} .
$$

We may assume that $\mathscr{A}^{* *}$ acts on the Hilbert space $\mathscr{H}$. Let $\psi^{\sim} \in \mathscr{T}(\mathscr{H})=$ $\mathscr{B}(\mathscr{H})_{*}$ (the predual of the bounded operators on $\mathscr{H}$ ) be an extension of $\psi$ such that $\left\|\psi^{\sim}\right\|=\|\psi\|[\mathbf{3 3}$, p. 156]. Then by Proposition 2.2 in [25], we have, for each $\tau$

$$
\begin{aligned}
1 & \geq\left\|\psi^{\sim}\right\|^{2} \geq\left\|P_{\tau} \psi^{\sim} P_{\tau}\right\|^{2}+\left\|P_{\tau} \psi^{\sim} Q_{\tau}\right\|^{2}+\left\|Q_{\tau} \psi^{\sim} P_{\tau}\right\|^{2}+\left\|Q_{\tau} \psi^{\sim} Q_{\tau}\right\|^{2} \\
& \geq\left\|P_{\tau} \psi P_{\tau}\right\|^{2}+\left\|P_{\tau} \psi Q_{\tau}\right\|^{2}+\left\|Q_{\tau} \psi P_{\tau}\right\|^{2}+\left\|Q_{\tau} \psi Q_{\tau}\right\|^{2},
\end{aligned}
$$

and so the inequality (1) follows. Now,

$$
\begin{aligned}
\left\|P_{\tau} \psi P_{\tau}\right\| & \geq\left|\psi\left(P_{\tau} X_{0} P_{\tau}\right)\right| \geq \operatorname{Re} \psi\left(P_{\tau} X_{0} P_{\tau}\right) \\
& =\operatorname{Re} \psi\left(X_{0}\right)-\operatorname{Re} \psi\left(P_{\tau} X_{0} Q_{\tau}\right)-\operatorname{Re} \psi\left(Q_{\tau} X_{0} P_{\tau}\right)-\operatorname{Re} \psi\left(Q_{\tau} X_{0} Q_{\tau}\right) \\
& \geq \delta_{0}-\|\psi\|\left(\left\|P_{\tau} X_{0} Q_{\tau}\right\|+\left\|Q_{\tau} X_{0} P_{\tau}\right\|+\left\|Q_{\tau} X_{0} Q_{\tau}\right\|\right) \\
& \geq \delta_{0}-3 \max \left\{\left\|X_{0} Q_{\tau}\right\|,\left\|Q_{\tau} X_{0}\right\|\right\} .
\end{aligned}
$$

But $X_{0} Q_{\tau}=X_{0}-X_{0} P_{\tau}$ and $Q_{\tau} X_{0}=X_{0}-P_{\tau} X_{0}$; consequently both of them converge to 0 in norm because $\left(P_{\alpha}\right)$ is an approximate identity. Thus, for $0<\eta<$ $\delta_{0}$, there is a $\tau_{0}$ such that if $\tau \geq \tau_{0}$ we have

$$
\left\|P_{\tau} X_{0} P_{\tau}\right\| \geq \delta_{0}-\eta \text {. }
$$

In particular, by (1), we have, for $\tau \geq \tau_{0}$,

$$
\alpha_{\tau}(\psi) \leq 3^{1 / 2}\left(1-(\delta-\eta)^{2}\right)^{1 / 2} .
$$

Now let $\xi>0$ be arbitrary. Since the weak* topology and the norm topology coincide on finite-dimensional subspaces, there is an $\alpha_{0}$ such that for all $\alpha, \beta \geq \alpha_{0}$,

$$
\left\|P_{\tau_{0}}\left(\varphi_{\alpha}-\varphi_{\beta}\right) P_{\tau_{0}}\right\|<\xi
$$

Consequently for all $\alpha, \beta \geq \alpha_{0}, \alpha \neq \beta$,

$$
\begin{aligned}
\left\|\varphi_{\alpha}-\varphi_{\beta}\right\| & \leq\left\|P_{\tau_{0}}\left(\varphi_{\alpha}-\varphi_{\beta}\right) P_{\tau_{0}}\right\|+\alpha_{\tau_{0}}\left(\varphi_{\alpha}\right)+\alpha_{\tau_{0}}\left(\varphi_{\beta}\right) \\
& <\xi+2(3)^{1 / 2}\left(1-\left(\delta_{0}-\eta\right)^{2}\right)^{1 / 2}
\end{aligned}
$$


by (2) and so

$$
\inf _{\alpha \neq \beta}\left\|\varphi_{\alpha}-\varphi_{\beta}\right\|<\xi+2(3)^{1 / 2}\left(1-\left(\delta_{0}-\eta\right)^{2}\right)^{1 / 2} .
$$

Since $\xi$ and $0<\eta<\delta_{0}$ are arbitrary we have

$$
\operatorname{sep}\left(\left(\varphi_{\alpha}\right)\right) \leq 2(3)^{1 / 2}\left(1-\left(\delta_{0}-\eta\right)^{2}\right)^{1 / 2}<\varepsilon_{1}<\varepsilon_{0} .
$$

This contradicts that $\operatorname{sep}\left(\left(\varphi_{\alpha}\right)\right)>\varepsilon_{0}$. This proves $\mathscr{A}^{*}$ must have property SUKK*

For the next few corollaries we refer the reader to $[33$, p. 157] for the relevant definitions and facts.

COROLlaRY 2. Let $\mathscr{A}$ be a dual $C^{*}$-algebra. Then $\mathscr{A}^{*}$ has property SUKK* and hence weak*-normal structure.

Let $\left\{\mathscr{A}_{i}: i \in I\right\}$ be a family of $C^{*}$-algebras. Then the $c_{0}$-direct sum of $\left\{\mathscr{A}_{i}: i \in I\right\}$ (this is called the $C(\infty)$-direct sum in [33, p. 157]), denoted by $\left(\sum_{i \in I} \bigoplus \mathscr{A}_{i}\right)_{0}$, consists of all those $x=\left(x_{i}\right), x_{i} \in \mathscr{A}_{i}$, such that for any $\varepsilon>0,\left\{i \in I:\left\|x_{i}\right\| \geq \varepsilon\right\}$ is finite. With the coordinatewise algebraic operations and the supremum norm, it is a $C^{*}$-algebra.

We proved in [24, Theorem 2] that $\mathscr{T}(\mathscr{H})$ has property SKK*, while Lennard $[\mathbf{2 5}$, Theorem 2.4] proved that it has property UKK*. The following include both of these results.

COROLlaRY 3. If $\mathscr{A}=\left(\sum_{\alpha \in I} \oplus \mathscr{C}\left(\mathscr{H}_{\alpha}\right)\right)_{0}$, where $\mathscr{C}\left(\mathscr{H}_{\alpha}\right)$ is the space compact operators on a Hilbert space $\mathscr{H}_{\alpha}$, then $\mathscr{A}^{*}$ has property SUKK*

Proof. Since each $\mathscr{C}\left(\mathscr{H}_{\alpha}\right)$ is a dual $C^{*}$-algebra this follows immediately from [33, 4(b), p. 157] and Corollary 2.

Let $G$ be a locally compact group. We define $C^{*}(G)$, the group $C^{*}$-algebra of $G$, to be the completion of $L_{1}(G)$ with respect to the norm

$$
\|f\|_{*}=\sup \left\|\pi_{f}\right\|
$$

where the supremum is taken over all nondegenerate representations $\pi$ of $L_{1}(G)$ as an algebra of bounded operator on a Hilbert space. Let $C(G)$ be the Banach space of bounded continuous complex-valued function on $G$ with the supremum norm. Denote the set of continuous positive definite functions on $G$ by $P(G)$, and the set of continuous functions on $G$ with compact support by $C_{00}(G)$. Define the Fourier-Stieltjes algebra of $G$, denoted by $B(G)$, to be the linear span of $P(G)$. The Fourier algebra of $G$, denoted by $A(G)$, is defined to be the closed linear span of $P(G) \cap C_{00}(G)$. Finally, let $\lambda$ be the left regular representation of $G$, i.e., for each $f \in L_{1}(G), \lambda(f)$ is the bounded operator in $\mathscr{B}\left(L_{2}(G)\right)$ defined on $L_{2}(G)$ by $\lambda(f)(h)=f * h$ (the convolution of $f$ and $h$ ). Then denote by $V N(G)$ to be the closure of $\left\{\lambda(f): f \in L_{1}(G)\right\}$ in the weak operator topology in $\mathscr{B}\left(L_{2}(G)\right)$. It is known that $C^{*}(G)^{*}=B(G)$ and $A(G)^{*}=V N(G)$. Furthermore, if $G$ is amenable (e.g., when $G$ is compact), then

$$
C^{*}(G) \cong \text { norm closure of }\left\{\lambda(f): f \in L_{1}(G)\right\} \subseteq V N(G) .
$$

We refer the reader to [14] for more details on these spaces. 
THEOREM 5. Let $G$ be an amenable locally compact group. Then the following are equivalent:

(1) $G$ is compact.

(2) $C^{*}(G)$ is dual.

(3) $B(G)$ has property SUKK*.

(4) $B(G)$ has property SKK*.

In particular, if $G$ is compact, then $B(G)$ has weak*-normal structure.

Proof. (1) $\Rightarrow(2)$. If $G$ is compact then $B(G)=A(G)$, and so $C^{*}(G)^{* *}=$ $A(G)^{*}=V N(G)$. Now by [5, Proposition 2.2, p. 345]

$$
C^{*}(G)=\{T \in V N(G): T \text { is compact }\} .
$$

Consequently $C^{*}(G)$ is an ideal in the enveloping von Neumann algebra $C^{*}(G)^{* *}$. Hence $C^{*}(G)$ is dual [33, p. 157].

$(2) \Rightarrow(3)$ follows from Corollary 2 .

(3) $\Rightarrow(4)$ is trivial.

$(4) \Rightarrow(1)$. Since $G$ is amenable, it follows from Lemma 7.2 in [22] that $A(G)$ has an approximate identity $\left(\phi_{\alpha}\right),\left\|\phi_{\alpha}\right\|=1$. By Theorem 3.2 in [22], the net $\left(\phi_{\alpha}\right)$ converges to the constant 1 function in the weak* topology of $B(G)$. By (4), then, the net must also converge to 1 in norm. Since $A(G)$ is a closed subspace of $B(G)$, the function 1 belongs to $A(G)$. By Theorem 3.34 in [14], $G$ must be compact.

\section{REFERENCES}

1. C. A. Akemann and M. E. Walter, Non-abelian Pontriagin duality, Duke Math. J. 39 (1972), 451-463.

2. D. E. Alspach, A fixed point free nonexpansive map, Proc. Amer. Math. Soc. 82 (1981), 423424.

3. J. Arazy, More on convergence in unitary matrix spaces, Proc. Amer. Math. Soc. 83 (1981), 44-48.

4. R. B. Burckel, Weakly almost periodic functions on semigroups, Gordon and Breach, New York, 1970.

5. C. Chou, A. T. Lau and J. Rosenblatt, Approximation of compact operators by sums of translations, Illinois J. of Math. 29 (1985), 340-350.

6. C. H. Chu, A note on scattered $C^{*}$-algebras and the Radon-Nikodym property, J. London Math. Soc. 24 (1981), 533-536.

7. D. F. Cudia, Rotundity, Proc. Sympos. Pure Math., vol. 7, Convexity, Amer. Math. Soc., Providence, R. I., 1963, pp. 73-97.

8. M. M. Day, Normed linear spaces, Springer-Verlag, New York, 1973.

9. J. Diestel, Geometry of Banach spaces-Selected topics, Lecture Notes in Math., vol. 485, Springer-Verlag, New York, 1975.

10. J. Diestel, Sequences and series in Banach spaces, Springer-Verlag, New York, 1984.

11. D. van Dulst and B. Sims, Fixed points of nonexpansive mappings and Chebyshev centres in Banach spaces with norms of type (KK), Banach Space Theory and its Applications, Proceedings Bucharest (1981), Lecture Notes in Math., vol. 991, Springer-Verlag, 1983.

12. D. van Dulst and V. de Valk, (KK)-properties, normal structure and fixed points of nonexpansive mappings in Orlicz sequence spaces, Canad. J. Math. 38 (1986), 728-750.

13. R. Engelking, General topology, PWN, Warsaw, 1977.

14. P. Eymard, L'algèbre de Fourier d'un groupe localement compact, Bull. Soc. Math. France 92 (1964), 181-236.

15. E. Granirer and M. Leinert, On some topologies which coincide on the unit sphere of the FourierStieltjes algebra $B(G)$ and of the measure algebra $M(G)$, Rocky Mountain J. Math. $11(1981)$, 459-472. 
16. E. Hewitt and K. Ross, Abstract harmonic analysis, Vol. I, Springer-Verlag, 1963.

17. R. Huff, Banach spaces which are nearly uniformly convex, Rocky Mountain J. Math. 10 (1980), 743-749.

18. W. A. Kirk, A fixed point theorem for mappings which do not increase distances, Amer. Math. Monthly 72 (1965), 1004-1006.

19. J. D. Knowles, On the existence of non-atomic measures, Mathematika 14 (1967), 62-67.

20. H. E. Lacey, The isometric theory of classical Banach spaces, Springer-Verlag, New York, 1974.

21. T. Landes, Normal structure and weakly normal structure of Orlicz sequence spaces, Trans. Amer. Math. Soc. 285 (1984), 523-534.

22. A. T. Lau, Uniformly continuous functionals on the Fourier algebra of any locally compact group, Trans. Amer. Math. Soc. 251 (1979), 39-59.

23. A. T. Lau and V. Losert, Complementation of certain subspaces of $L_{\infty}(G)$ of a locally compact group, Pacific J. Math. (to appear).

24. A. T. Lau and P. F. Mah, Quasi-normal structures for certain spaces of operators on a Hilbert space, Pacific J. Math. 121 (1986), 109-118.

25. Chris Lennard, $C_{1}$ is uniformly Kadec-Klee*, Proc. Amer. Math. Soc. (to appear).

26. T. C. Lim, Asymptotic centres and nonexpansive mappings in some conjugate spaces, Pacific J. Math. 90 (1980), 135-143.

27. V. Losert and H. Rindler, Uniform distribution and the mean ergodic theorem, Invent. Math. 50 (1978), 65-74.

28. Paul Milnes, On the extension of continuous and almost periodic functions, Pacific J. Math. 56 (1975), 187-193.

29. I. Namioka and R. R. Phelps, Banach spaces which are Asplund spaces, Duke Math. J. 42 (1975), 735-750.

30. J. P. Pier, Amenable locally compact groups, Wiley, New York, 1984.

31. Z. Semandeni, Banach spaces of continuous functions, PNW, Warsaw, 1971.

32. M. A. Smith and B. Turret, A reflexive LUR Banach space that lacks normal structure, Canad. Math. Bull. 28 (1985), 492-494.

33. M. Takesaki, Theory of operator algebras I, Springer-Verlag, New York, 1979.

Department of Mathematics, University of Alberta, Edmonton, Alberta, CANADA T6G 2G1

Department of Mathematical SCiences, LAKehead University, Thunder Bay, ONTARIO, CANADA P7B 5E1 